Kieler Beiträge zur Filmmusikforschung, 2, 2008 / 7

\title{
Gender-Konstruktion durch Filmmusik. Eine analytische Betrachtung am Beispiel der Vertonung von Frauenfiguren in Filmen von Alfred Hitchcock und im neueren Frauenfilm
}

Claudia Bullerjahn (Gießen)

\section{Einleitung}

Gerade in den letzten Jahren haben sich Wissenschaftler vermehrt dem vordem stiefmütterlich behandelten Thema Filmmusik zugewandt, jedoch bisher kaum unter dem Genderaspekt. Ausnahmen bilden die Publikationen von Kathryn Kalinak (1982), Caryl Flinn (1986, 1992), Eva Rieger (1996) und Anahid Kassabian (2001).

Insbesondere der auf ein Massenpublikum angelegte Hollywoodfilm ist gekennzeichnet durch typisierte Identitäten und habitualisierte Aktivitäten von Männern und Frauen. Es stellt sich nun die Frage, ob Filmmusik diese geschlechtsspezifischen Identitäten und Aktivitäten unterstützt oder konterkariert. Werden überhaupt unterschiedliche musikalische Mittel angewandt, um Männer und Frauen zu beschreiben? Spielfilme sind immer auch ein Spiegel gesellschaftlicher Veränderungen im Geschlechterverhältnis. Spiegeln sich diese Veränderungen auch in der Filmmusik wider?

Aufgrund der vorgegebenen Begrenzung des Umfangs kann in dem vorliegenden Artikel nur exemplarisch vorgegangen werden, und es wird sicherlich nicht gelingen, diese Fragen abschließend für jeden denkbaren Film zu klären. Zunächst werden die filmmusikalischen Profile der kulturellen Kategorien >männlich` und >weiblich` vorgestellt und Erklärungen für ihre Entstehung und hartnäckige Verwendung angeboten. Im Anschluss werden Hitchcocksche Frauenfiguren hinsichtlich ihrer Vertonung untersucht, wobei die Filme Rebecca und Vertigo explizit im Mittelpunkt stehen, deren Gemeinsamkeit eine schon Verstorbene mit gleichwohl noch sehr lebendigem musikalischen Eigenleben ist. Im Vergleich hierzu werden die neueren Filme Out of Rosenheim, Fried Green Tomatoes und Thelma \& Louise betrachtet, in denen Freundschaften zwischen zwei recht verschiedenen, jedoch gleichermaßen sich von ihren Männern emanzipierenden Frauen eine bedeutende Rolle spielen und in denen ein gewandeltes Frauenbild möglicherweise auch mit 
andersartigen Vertonungen einhergeht. ${ }^{1}$ Zusammenfassende und ausblickende Anmerkungen beschließen die Ausführungen.

\section{Filmmusikalische Profile der kulturellen Kategorien >männlich` und >weiblich}

»Musik ist physikalisch neutral. Andererseits kann Musik nur serlebt〈, also in Bezug zum eigenen Leben gestellt werden, wenn sie in ein vorgefertigtes Bezugssystem von Stereotypen und Konventionen passt.«(Rieger 1996, 12f).

Die kulturelle Fähigkeit, eine spezifische Filmmusik in spezifischer Weise zu interpretieren, wird von allen Individuen geteilt, die den gleichen kulturell-medialen Einflüssen ausgesetzt sind. Diese Fähigkeit ist so selbstverständlich, dass kaum bewusst darüber nachgedacht wird. Auch sind die meisten Personen nicht in der Lage $\mathrm{zu}$ verstehen und $\mathrm{zu}$ benennen, wie und warum musikalische Kommunikation tatsächlich funktioniert. Dies hat unter anderem damit zu tun, dass die verschiedenen musikalischen Wissensbereiche an spezifische Bildungsinstitutionen gebunden sind, wie z.B. die Musikausübung an Musikhochschulen und Konservatorien, die Musikanalyse und -theorie an historische musikwissenschaftliche Institute und die Interpretation der Beziehung zwischen Kultur, Gesellschaft und Individuum an systematische musikwissenschaftliche Institute sowie angrenzende Sozial-, Medien- und Kulturwissenschaften. Ein vollständiges Verständnis von Filmmusik wird dem Laien durch diese Aufteilung musikalischen Wissens versperrt, was ihm die Macht nimmt, Manipulationen zu durchschauen oder gar alternative filmmusikalische Kommunikation zu entwerfen (vgl. Tagg 2006, 166-168).

In welcher Weise musikalische Stereotypen und Konventionen in audiovisuellen Medien eine Rolle spielen und welche Bedeutungen somit durch Filmmusik kommuniziert werden, untersuchten Philip Tagg und Bob Clarida empirisch (Tagg/Clarida 2003; Tagg 2006, 172-178). Sie spielten über 600 Probanden zehn kurze, rein instrumentale Titelthemen aus Film und Fernsehen vor und forderten sie auf, das, was sie innerlich sahen oder sich beim alleinigen Hören vorstellten, aufzuschreiben. Es zeigte sich trotz der Methode der freien Induktion (und nicht Multiple Choice) eine signifikante Konsistenz der Antworten und recht geringe Streuung über die verschiedenen Melodien. Kriterien für die Klassifikation innerhalb der qualitativen Inhaltsanalyse konnten aus Systematisierungen der Funktionen nach Filmmusik nach Zofia Lissa (1965, 115-256) und Archivmusik- und Kinothekenklassifikationen (etwa Rappée 1970) sowie durch den musikalischen Alltagsgeschmack, den man als Mitglied unserer Kultur erwirbt, und einige hermeneutische Fertigkeiten gewonnen werden. Besonders überraschend war die klare Trennung der musikalischen und paramusikalischen Profile der kulturellen Kategorien >männlich` und >weiblich . Bei fünf Melodien wurden eher männliche, bei den anderen fünf eher weibliche Personen assoziiert (vgl. Tab. 1).

$1 \mathrm{Zu}$ den filmmusikalischen Fachbegriffen und praktischen Empfehlungen für die Filmmusikanalyse vgl. Bullerjahn 2001 und 2005 . 
Kieler Beiträge zur Filmmusikforschung, 2, 2008 / 9

Tab. 1: \Männliche` und `weibliche〈 Melodien (Tagg 2006, Abb. 6.2)

\begin{tabular}{|llllll|}
\hline Melodie & männlich & weiblich & Gesamt & männlich\% & weiblich\% \\
\hline ,männliche' Melodien & & & & & \\
\hline The VIRGINIAN & 36,9 & 0,7 & 38,0 & 98,2 & 1,8 \\
\hline SPORTSNIGHT & 16,3 & 1,6 & 18,0 & 90,9 & 9,1 \\
\hline Owed TO ,G' & 22,1 & 1,4 & 23,0 & 94,1 & 5,9 \\
\hline MIAMI VICE & 11,4 & 1,0 & 12,0 & 70,0 & 30,0 \\
\hline ,weibliche' Melodien & & & & & \\
\hline Dream OF OLWEN & 0,5 & 12,7 & 13,0 & 3,7 & 96,3 \\
\hline RoMeO \& JULIET & 4,8 & 17,2 & 22,0 & 21,9 & 78,1 \\
\hline EMMERDALE FARM & - & 4,1 & 4,1 & - & 100,0 \\
\hline SAYONARA & 4,1 & 13,1 & 17,0 & 23,8 & 76,2 \\
\hline
\end{tabular}

Einige der wesentlichen musikalischen Unterschiede zwischen den `männlichen` und `weiblichen` Melodien werden in Tab. 2 zusammengefasst. Durch die Analyse dieser Melodien konnten recht typische musikalische Charakteristika hinsichtlich Genre und Stil herausgearbeitet werden und somit die recht hohe Stabilität des filmmusikalischen Bedeutungssystems und der hohe Grad an Konstistenz, wenn auch keiner der Befragten komplett identische Antworten abgab.

\section{Tab. 2: Einige musikalische Charakteristika von >männlichen $\$ und $>$ weiblichen $<$ Melodien}

(Tagg 2006, Tab. 6.3)

\begin{tabular}{|lll|}
\hline musikalische Charakteristika & männliche Melodien & weibliche Melodien \\
\hline durchschnittliches Tempo & $109 \mathrm{bpm}$ & $83 \mathrm{bpm}$ \\
\hline $\begin{array}{l}\text { Generelle Geschwindigkeit der } \\
\text { schnellsten Noten }\end{array}$ & c. 400 & c. 180 \\
\hline Phrasenlänge & kurz & \\
\hline Phrasierung & Stakkato & lang \\
\hline wiederholte Noten & häufig & Legato \\
\hline Dynamikwechsel & keine & keine \\
\hline Basslinie & aktiv und kantig & einige \\
\hline Offbeats und Synkopierungen & häufig & recht statisch \\
\hline Instrumentation der Melodie & E-Gitarre, Gitarren-Synthesizer, & selten \\
& Trompete, Xylophon & Streicher, Flöte, Mandoline, \\
\hline Instrumentation der Begleitung & Gitarren-Riffs und -Geklimpere, & Oboe, Klavier \\
& Blechbläsereinwürfe, Sequencer- & Streicher, Klavier, Holzbläser, \\
& Synthesizer, Schlagzeug & Slechbläser, kein \\
\hline tonales Idiom & Rock, häufig (abgemilderter) Jazz & Klassik, häufig Romantik \\
\hline
\end{tabular}

Tab. 3 fasst einige Gender-Polaritäten zusammen, die eine Re-Analyse der Daten durch Kassabian ergab.

Insgesamt betrachtet wurden Frauen zweimal häufiger als Männer mit der freien Natur assoziiert, siebenmal häufiger als Männer mit den Jahreszeiten oder dem Wetter in Beziehung gesetzt und zwölfmal häufiger als Männer in ländlichen Settings gesehen. Frauen wurden außerdem wesentlich wahrscheinlicher als Männer mit Stille und Ruhe in Verbindung gebracht, 25 Prozent häufiger mit Liebe assoziiert und öfter als traurig, 


\section{Kieler Beiträge zur Filmmusikforschung, 2, 2008 / 10}

melancholisch oder nostalgisch eingeschätzt. Darüber hinaus erschienen sie niemals als ungesellig oder gar Waffen tragend. Ganz im Gegensatz dazu wurden Männer neunmal häufiger als Frauen mit städtischen Räumen assoziiert, neunmal häufiger mit Innenräumen, zwanzigmal häufiger mit Autos und 35mal häufiger mit Clubs und Kneipen. Es war außerdem zu 33 Prozent wahrscheinlicher, dass man sie in öffentlichen Veranstaltungen jedweder Art antraf, sie waren zu fünfzig Prozent stärker als Frauen, sie wurden niemals isoliert oder ausgeschlossen dargestellt und sie waren niemals traurig. Selbstverständlich konnten sie ungesellig und Waffen tragend sein. Zwar sind die geäußerten Genderassoziationen visuell und verbal inkongruent, jedoch beinhalten sie eine hohe emotionale, taktile und gestische Kongruenz (Tagg 2006, 176f).

\section{Vertonung Hitchcockscher Frauenfiguren}

Bekanntermaßen fand schon im ausgehenden 18. und 19. Jahrhundert eine ideologische Festigung der Geschlechterrollen statt: Mann und Frau wurden unterschiedliche Charaktereigenschaften zugewiesen, was sich auch in der bürgerlichen Musik widerspiegelte. Insbesondere in der Oper dominierten zwei musikalische Weiblichkeitsdarstellungen: Die bürgerlich-eingegrenzte Weiblichkeit war charakterisiert durch ununterbrochene, gebundene Melodik, regelmäßige Rhythmik und Periodik, geringen Ambitus, melismatische Stimmbehandlung, kleinschrittige Intervalle und Seufzervorhalte. Die gefährliche, erotisch attraktive Weiblichkeit war dagegen gekennzeichnet durch extreme Intervallsprünge, unterbrochene, abgehackte Melodik, unregelmäßige Rhythmik, Molltonart, Chromatik und Dissonanzen sowie absteigende Bässe (Rieger 1996, 15f). Beispielsweise finden sich in den Opern Die Zauberflöte und Carmen beide musikalischen Weiblichkeitsdarstellungen.

Einigen Stummfilmkomponisten, unter anderem dem ausgebildeten Opernsänger Gottfried Huppertz (18971937), ist es zu verdanken, dass diese gegensätzlichen Weiblichkeitsdarstellungen auch Eingang in den Spielfilm fanden: In seiner Originalmusik zu dem zweiteiligen Stummfilm Die Nibelungen (Deutschland 1924) vom Regisseur Fritz Lang arbeitet er mit Personen-Leitmotiven und gestaltet durch sie die beiden dominierenden Frauengestalten zunächst recht gegensätzlich. Kriemhilds erstes Thema charakterisiert diese durch Instrumentation (Holzbläser), Dynamik (piano) und Rhythmik (Synkopen) als sanftmütige, passive und unsichere Person. Wenn im Film jedoch ihre charakterliche Veränderung offenbar wird - sie schwört, den feigen Mord an ihrem Gatten Siegfried zu rächen, und wird somit zur grausamen und aktiven Person -, wird das erste Thema nahezu vollständig von einem zweiten Thema abgelöst, das sie durch chromatische Linienführung, einen übermäßigen Quartschritt und synkopierten sowie punktierten Rhythmus als böse Frau kennzeichnet. Brunhild, die stolze und starke Königin von Island, bildet den Gegenpol zu der noch arglosen und naiven Kriemhild des ersten Filmteils und wird in ihrer Ungeduld und Heftigkeit durch eine triolische Bassfigur in der Begleitung und einen von tremolierenden Streichern gespielten Abgang im zweiten Teil ihres 
Themas charakterisiert. Verminderte Dreiklänge und Chromatik prägen zusätzlich Brunhilds Bild (Fabich 1993, 205-208).

Tab. 3 Gender-Polaritäten gemäß den Dichotomien `Natur/Kultur`, >beweglich/unbeweglich` und >privat/öffentlich` (Kassabian 2001, Zusammenfassung der Tab. 1, 2 u. 3)

\begin{tabular}{|lll|}
\hline Kategorie & \multicolumn{2}{l|}{ durchschnittliches prozentuales Auftreten } \\
\hline & weiblich & männlich \\
\hline Natur/Kultur & & \\
\hline draußen & 0,96 & 0,42 \\
\hline drinnen & 0,84 & 8,39 \\
\hline Clubs & 0,21 & 7,11 \\
\hline abgelegene Orte & 0,67 & 0,00 \\
\hline Autos & 0,46 & 9,23 \\
\hline ländlich & 27,38 & 2,35 \\
\hline städtisch & 2,28 & 16,87 \\
\hline Wetter & 7,64 & 1,20 \\
\hline Jahreszeiten & 4,21 & 0,30 \\
\hline beweglich/unbeweglich & & \\
\hline Reflexion & 4,29 & 0,17 \\
\hline Stillstand & 2,77 & 0,75 \\
\hline Dynamik & 3,73 & 11,30 \\
\hline kulturell aufstrebend & 0,00 & 4,82 \\
\hline Schicksal & 4,73 & 0,00 \\
\hline widerwillig & 3,92 & 0,00 \\
\hline festgelegtes Verhalten & 1,54 & 0,33 \\
\hline widersprüchlich & 0,46 & 3,52 \\
\hline übertragbar & 0,13 & 7,89 \\
\hline privat/öffentlich & & \\
\hline Ruhe & 4,42 & 0,03 \\
\hline Stärke & 0,09 & 1,27 \\
\hline Ungeselligkeit & 0,00 & 1,65 \\
\hline Liebe & 21,79 & 0,88 \\
\hline Waffen & 0,00 & 0,30 \\
\hline festlich & 0,21 & 2,67 \\
\hline präsentierbar & 0,36 & 11,87 \\
\hline Militär & 0,04 & 2,84 \\
\hline & & \\
\hline
\end{tabular}

In zahlreichen frühen Tonfilmen Hollywoods wurden diese gegensätzlichen Weiblichkeitsdarstellungen übernommen (Rieger 1996, 19f), was nicht verblüfft, wenn man sich den großen europäischen Einfluss auf Kunstmusik und Film vergegenwärtigt, der durch die Emigration einer Vielzahl von europäischen Komponisten und deren Engagement im Filmgeschäft zeitweilig noch verstärkt wurde. Am Beispiel zweier Filme von Alfred Hitchcock aus verschiedenen Schaffensperioden soll nun demonstriert werden, dass die Vertonung von Frauenfiguren noch lange in dieser Tradition stattfand. Zur Konstruktion der Frau als voyeuristisches Objekt haben durch die feministische Filmtheorie aufgedeckte spezifische filmische Codes 


\section{Kieler Beiträge zur Filmmusikforschung, 2, 2008 / 12}

beigetragen (Rieger 1996, 19). Gerade in Filmen Hitchcocks begegnet man der ohnehin im erzählenden Kino häufigen Zurschaustellung der Frau und insbesondere ihres Körpers »als Ort der Sexualität und als Gegenstand des Begehrens« (Rieger 1996, 104). Dies geschieht jedoch hier oft in ironisch gebrochener oder vielschichtiger Weise. Hitchcock baut im Wesentlichen auf den Kontrast zweier immergleicher Frauentypen: zum einen die den Mann gefährdende, attraktive, idealisierte Blondine, zum anderen das den Zwängen einer patriarchalen Gesellschaft ausgelieferte, wenig attraktive Hausmütterchen. Hinzu treten teilweise noch diabolische, den blonden Vamp unterstützende Frauengestalten. Alle Frauengestalten erleiden in der Regel Grausames innerhalb des narrativen Geschehens, werden bedroht, verfolgt, vergewaltigt oder sogar getötet.

»Die Gleichsetzung von Liebe mit dem (möglichst verhallten) Klang vieler Violinen ist ein HollywoodStereotyp par excellence« (Rieger 1996, 104). Häufig wird das Liebesthema ausschließlich mit der weiblichen Hauptperson verbunden, was der Ambivalenz entspricht, die in der westlichen Zivilisation der Frau entgegengebracht wird: »[...] einerseits ihre Zuweisung und Beschränkung auf die Emotionen, andererseits die Betonung ihrer >natürlichen` Liebesfähigkeit« (Rieger 1996, 115). Dies entspricht der kulturellen Kategorie `weiblich $\prec$ hinsichtlich ihrer musikalischen und paramusikalischen Profile, wie sie sich in der oben besprochenen Studie von Tagg und Clarida zeigten.

\section{RebecCa (USA 1940)}

Hitchcocks erster Hollywood-Spielfilm REBECCA beinhaltet drei zentrale Frauengestalten, wobei das Besondere darin besteht, dass die titelgebende Rebecca de Winter schon vor längerer Zeit verstorben ist und im Film nur indirekt über ihre persönlichen Habseligkeiten (mit Initialen gekennzeichnetes Adressbuch, Kleidung, Mobiliar, gemaltes Porträt etc.) visuell in Erscheinung tritt. Filmmusik erhält hierdurch eine herausragende Rolle, denn nur über ihr Leitmotiv kann sich Rebecca akustisch einfinden und emotionale Präsenz erhalten. Der Komponist Franz Waxman (1906-1967) folgt bei Rebeccas Leitmotiv der Typisierung der bösen, verrückten oder begehrenden Frau, wie sie ihm als in der Kunstmusik durch ein Studium in Dresden und Berlin ausgebildetem Europäer aus zahlreichen Opern des 19. Jahrhunderts vertraut gewesen sein muss. Eine sich schlängelnde Melodik, angereichert durch Chromatik sowie Umspielungen von Tritonus und verminderter Septime, über einem harmonisch unsicheren Grund in zumeist unangenehmer Klangfarbe, elektronisch erzeugt vom Novachord, vermittelt negativ-böse und zugleich faszinierende Klänge (vgl. NB 1 a). Das Leitmotiv der neuen Mrs. de Winter ist dagegen schlicht, rhythmisch gleichmäßig, diatonisch, von geringem Ambitus, nicht zielgerichtet und dünn instrumentiert (vgl. NB 1 b). ${ }^{2}$ Überraschenderweise spiegelt sich ihre in der Handlung deutlich werdende Entwicklung zur reifen, Initiative ergreifenden Frau nicht

2 Eine ähnliche Dichotomisierung des Weiblichen komponierte Franz Waxman übrigens für den Hitchcockfilm The PARADINE CASE (USA 1947) (vgl. ebd., S. 115-119). Auch der Hitchcockfilm Stagefright (USA 1950) mit der Musik von Leighton Lucas (19031982) blieb diesem Muster treu. 


\section{Kieler Beiträge zur Filmmusikforschung, 2, 2008 / 13}

musikalisch in den Variationen ihres Leitmotivs wider. Ergänzt wird ihr Leitmotiv zwar durch ein Liebesthema, jedoch ist dieses mehr ihrem Ehemann zugeordnet und in seinem durch Oktavsprünge weit ausgreifenden Charakter und seine aufwärtsgerichtete Dur-Melodik positiv vorwärtsweisend und somit gegensätzlich zu Rebeccas Leitmotiv angelegt (vgl. NB 1 c). Das Leitmotiv der Haushälterin Mrs. Danver umrahmt den Tritonus, seit dem Barock ein Sinnbild des bösen Omens und drohenden Unheils (vgl. NB 1 d), was eine durchaus treffende musikalische Charakterisierung ist, denn schließlich ist sie es, die am Ende den Landsitz Maxim de Winters in Schutt und Asche legt und gewissermaßen als Strafe in den Flammen selbst umkommt.

(a)
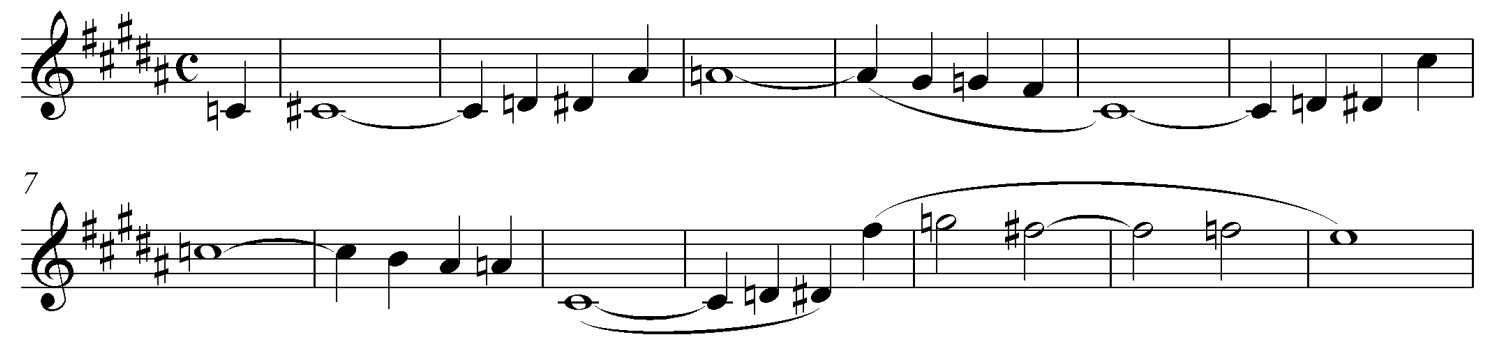

(b)

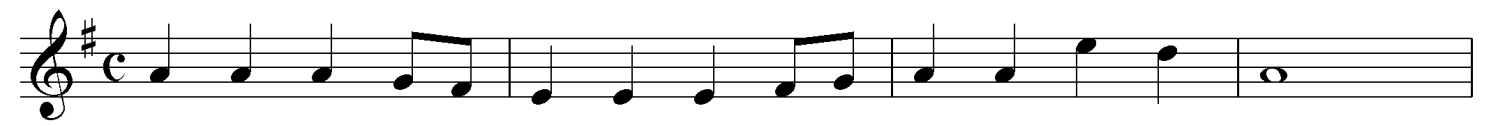

(c)
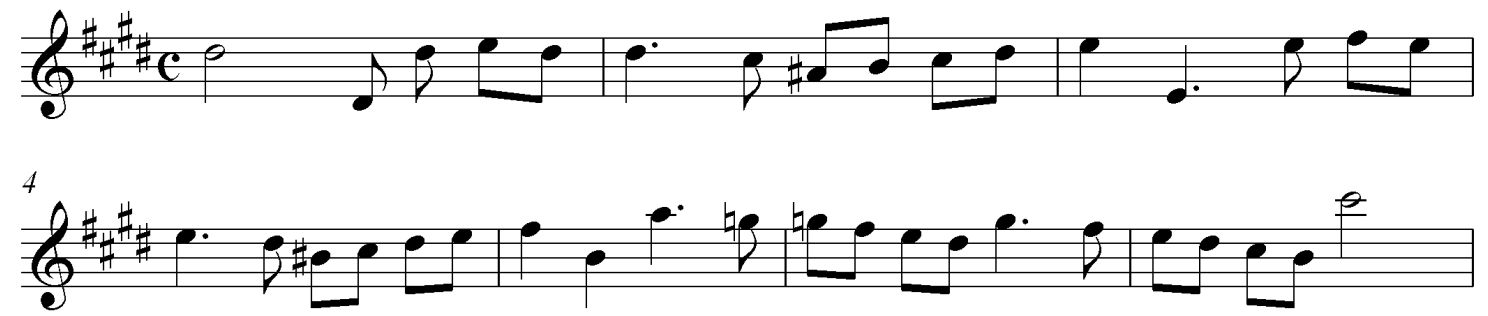

(d)

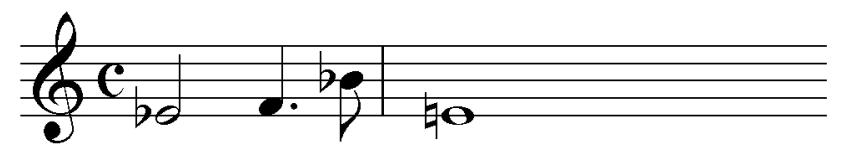

NB 1:

Leitmotive aus Rebecca: (a) Rebeccas Leitmotiv, (b) Leitmotiv der neuen Mrs. de Winter, (c) Liebesthema, (d) Leitmotiv der Haushälterin Mrs. Danver ${ }^{3}$

Rebecca ist von Anfang an eine Bedrohung für das junge Eheglück der neuen Mrs. de Winter, denn der Zuschauer erhält durch die Narration auf visueller und sprachlicher Ebene zunächst den Eindruck, dass Rebecca durch ihre Schönheit, ihren distinguierten Geschmack und die kompetente Führung ihres Hauspersonals, welches ihr zum Teil über den Tod hinaus treu ergeben ist, die ideale Ehefrau für Maxim de

3 Die melodischen Transkriptionen wurden aus Rieger 1996, S. 251, übernommen. 


\section{Kieler Beiträge zur Filmmusikforschung, 2, 2008 / 14}

Winter verkörperte, deren Verlust er lange betrauerte. Somit schneidet Rebecca im Vergleich mit der unsicheren, naiven und tollpatschigen neuen Ehefrau stets gut ab, denn erst im späteren Verlauf des Films erfährt diese - und somit auch der Zuschauer - von Rebeccas bösartigen Charakter und dass sie ihren Ehemann nie liebte und stets betrog. Allerdings ahnt der aufmerksame Zuhörer dies von Anfang an, denn Rebeccas Leitmotiv verkörpert in stereotyper Weise das musikalisch Böse, das zugleich ja immer auch faszinierender als das häufig langweilige Gute ist: »Der Einsatz von Hall und elektronischen Klängen [...] ruft unangenehme Assoziationen hervor. Doch lassen die Chromatik und die Aufschwünge auch die Faszination des Verführerisch-Sinnlichen zu« (Rieger 1996, 77).

\section{VERTIGO (USA 1958)}

Der Film VerTIGo stellt eine Auseinandersetzung mit der Unerfüllbarkeit erotischer Sehnsüchte dar: Eine tragische Liebesgeschichte wird mit der Hinterfragung des Phänomens der romantischen Liebe verknüpft. Der vorzeitig pensionierte Polizist Scottie leidet unter Höhenangst, die »symbolisch für die Angst [steht], sich einem anderen Menschen bedingungslos hinzugeben und dabei die Orientierung zu verlieren« (Rieger 1996, 176). In Scotties Sehnsucht, eine Tote lebendig machen bzw. eine Lebende an das Vorbild einer Toten anpassen zu wollen, verschränken sich Orpheus- und Pygmalion-Mythos. Darüber hinaus thematisiert der Film männlichen Voyeurismus - schließlich ist die Beschattung einer Frau zentral für die Handlung - und Fetischismus.

Entgegen seiner sonstigen Gewohnheit arbeitet der Komponist Bernard Herrmann (1911-1975) mit vergleichsweise prägnanten Leitmotiven, die vornehmlich den zentralen Frauengestalten zugedacht sind: Madeleine, der zu beschattenen Ehefrau eines Bekannten, ist das Liebesthema zugeordnet (vgl. NB 2 a), das mit seiner legato-Melodie in den sordino-Streichern und den Seufzermotiven eine traditionelle musikalische Beschreibung des Weiblichen liefert, jedoch durch die zusätzliche Verwendung des Tritonus ihr tragisches Schicksal vorwegnimmt. Es kennzeichnet die Wahrnehmung Madeleines durch Scottie und liefert damit nicht nur eine Illustration seines Zustands der Obsession, sondern auch die Basis für eine Einfühlung des Zuschauers. Midge, die mütterliche Jugendfreundin Scotties, wird dagegen nur sparsam durch tiefe Streicher musikalisiert und mit biederer klassischer Musik in Zusammenhang gebracht. Bei Carlotta, der vorgeblichen Vorfahrin Madeleines, deren Gemälde sie im Museum anscheinend stundenlange Besuche abstattet, da sie sich angeblich einbildet, von ihrem Geist besessen zu sein, wird ihre südamerikanische Herkunft durch einen Habañera-Rhythmus in einem ansonsten „typisch weiblichen Thema“ angedeutet (vgl. NB 2 b). Für die Verkäuferin Judy, die im Auftrag von Madeleines Ehemann Madeleine mimte und dafür sorgte, dass Scottie den Eindruck gewann, dass Madeleine sich während einer Beschattungsaktion vor seinen Augen das Leben nahm, werden nur an einer Filmstelle volkstümliche Klänge im 6/8-Takt eingesetzt, bevor sie durch Änderung von Haarfarbe und Kleidung auf Wunsch von Scottie in Madeleine (zurück)verwandelt wird und 
eine Variation des hier bezeichnenderweise an Wagners Liebestod aus Tristan und Idolde gemahnenden Liebesthemas zugeordnet bekommt.

(a)
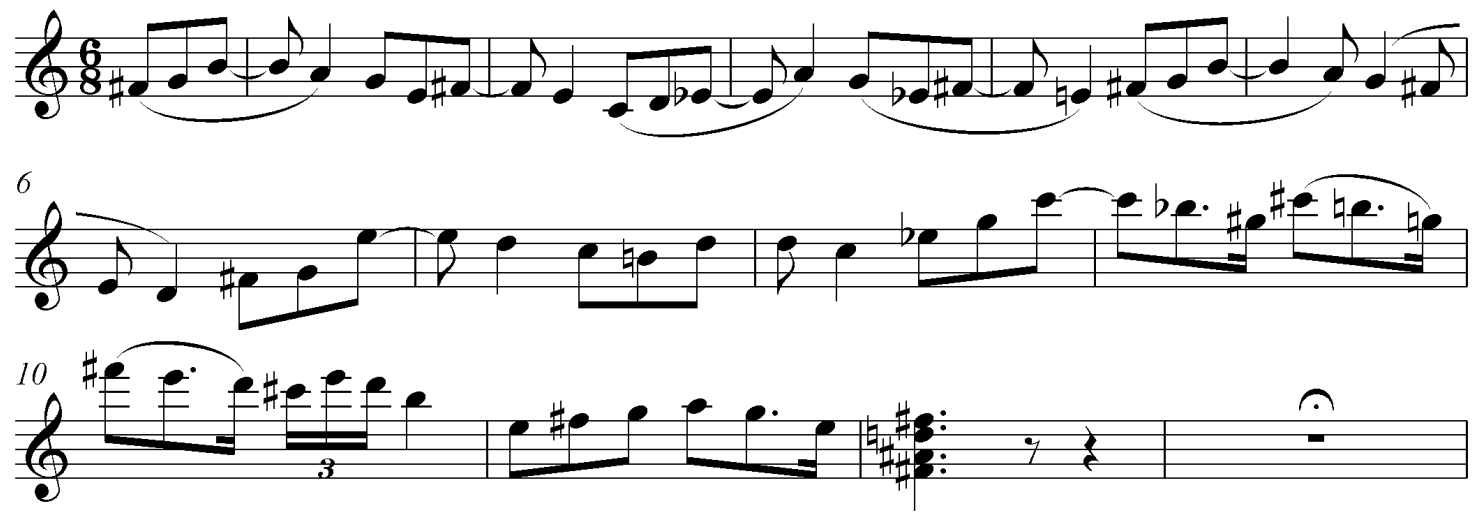

(b)
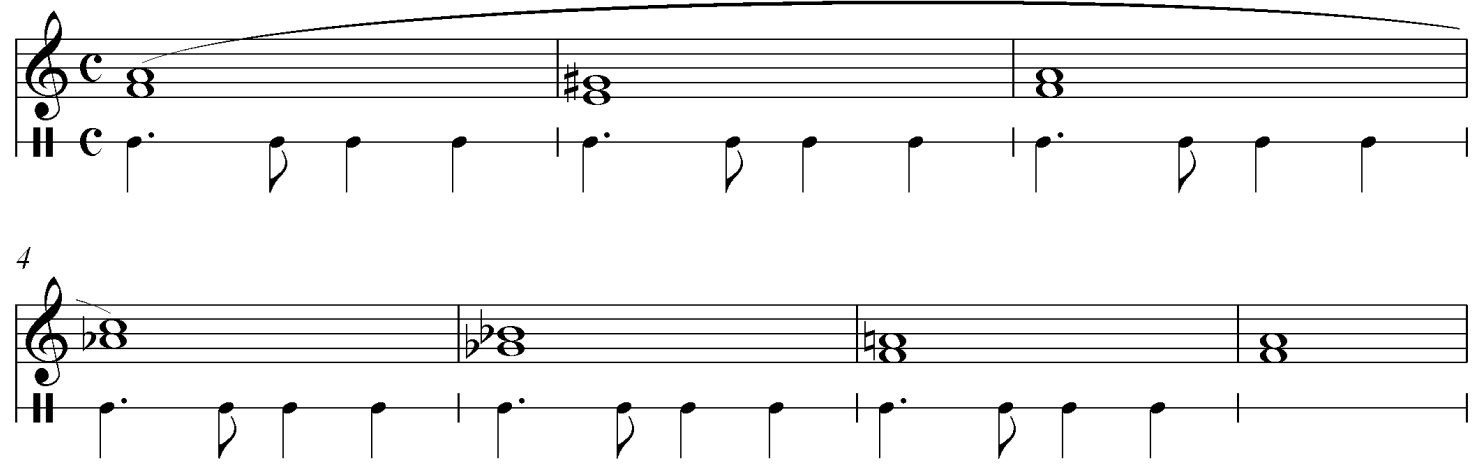

(c)

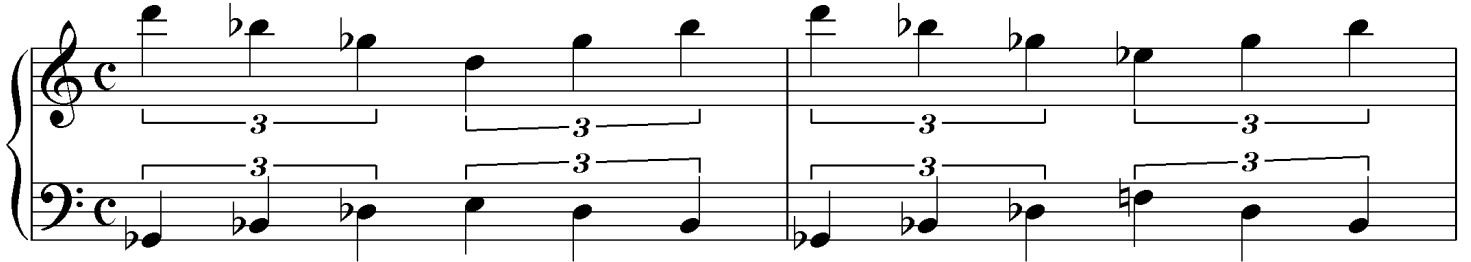

NB 2:

\section{Leitmotive aus Vertigo: (a) Liebesthema, das Madeleine und der verwandelten Judy zugeordnet ist, (b) Carlottas Thema, (c) Vertigo-Thema aus dem Präludium ${ }^{4}$}

Für Judy, die Scotties Liebe erwidert, gibt es keinen Ausweg aus der Falle. Da sie den Betrug an Scottie mit $\mathrm{zu}$ verantworten hat und in das Mordkomplott verwickelt ist, ist sie schuldbeladen und kommt bei einem Streit mit Scottie unter unglückseligen Umständen durch einen Sturz vom Kirchturm auf die gleiche Weise um wie das zuvor von ihr gemimte Opfer. Die im Vorspann prägnant abstrakt visualisierte Spirale, die an verschiedenen Stellen des Films wieder auftaucht (z.B. Haarknoten Madeleines, Turmtreppe), steht einerseits für Scotties aus der Höhenangst resultierenden Schwindel (engl. vertigo), andererseits für die Endlosigkeit 


\section{Kieler Beiträge zur Filmmusikforschung, 2, 2008 / 16}

und Unerfüllbarkeit romantischer Sehnsucht. Im Eingangsmotiv des Präludiums, das beim Auftreten von Scotties Höhenangst immer wieder ertönt - aber auch bei Judys sschwindelerregender Verwandlung in Madeleine - sorgen fehlende Abkadenzierung, fehlende tonale Ausrichtung und zwei unaufgelöste, auf- und abwärts gebrochene übermäßige Dreiklänge ohne Bassstütze für ein Äquivalent auf musikalischer Ebene (vgl. NB 2 c). Letztendlich ist es die aus Höhenangst und Obsession resultierende Schwäche Scotties, die eine echte Liebesbeziehung unmöglich macht.

\section{Musikalische Charakterisierung von Frauen im neueren Spielfilm}

Anahid Kassabian arbeitet in ihrer Monographie heraus, dass das zeitgenössische Hollywoodkino von zwei filmmusikalischen Hauptansätzen bestimmt werde, die tendenziell auf unterschiedliche Weise Identifikationsprozesse in Gang setzten: zum einen speziell für den jeweiligen Film komponierte Filmmusik in klassischer Hollywoodtradition, die für integrierende Identifikationen (`assimilating identifications $\measuredangle$ ) sorge, und zum anderen aus zumeist schon vorher existierenden Songs kompilierte Filmmusik, die angliedernde Identifikationen (>affiliating identifications $\triangleleft$ ) nach sich ziehe (Kassabian 2001, 2f). Speziell komponierte Filmmusik sorge in standardisierter, häufig auch klischeehafter Weise und damit recht rigide kontrolliert in ihrer Bedeutungsproduktion dafür, dass Filmrezipienten in sozial und historisch unvertraute Positionen hineingezogen werden und sich trotz fehlenden Bezugs zur eigenen Lebensgeschichte dennoch mit einer spezifischen Person identifizieren könnten. Bei kompilierter Filmmusik dagegen brächten Filmrezipienten durch die verwendeten Songs aus der Erinnerung aufgerufene, wenig kontrollierbare, vielfältige externe Assoziationen in die Filmnarration mit ein, was die Möglichkeit zur Identifikation mit verschiedenen Filmfiguren offen halte. Selbstverständlich existieren auch Vermischungen beider filmmusikalischer Ansätze. Die neue Vielfältigkeit von Filmmusik wurde nach Kassabian u.a. durch die Aufnahme neuer Filmthemen wie Gender- und Rassenproblematiken verursacht.

Nach Kassabian nahm während der 1980er Jahre die Anzahl der Filme mit Popmusik-Soundtracks signifikant zu, davon die meisten kompiliert aus Rock- und Popsongs (Kassabian 2001, 61). Ein Grund hierfür ist sicherlich der potentielle Gewinn aus dem Verkauf des Soundtrackalbums. Dennoch handelt es sich keineswegs um eine neue Marketingstrategie, wie z.B. die Filme The Graduate (USA 1967, Mike Nichols) mit Songs von Simon \& Garfunkel, EAsy Rider (USA 1969, Dennis Hopper) mit diversen Rocksongs, Harold and Maude (USA 1971, Hal Ashby) mit Songs von Cat Stevens bzw. American Graffiti (USA 1973, George Lucas) mit diversen Rock-'n'-Roll-Songs der frühen 1960er Jahre belegen. Themensongs sowie Musik und insbesondere Songs als einziges akustisches Element einer Szene erhalten immer hohe Aufmerksamkeit im Vergleich zu Musik im Hintergrund von geräuschhafter Handlung oder Dialogen (Kassabian 2001, 52). Gerade Filme mit starken weiblichen Charakteren werden nach Einschätzung Kassabians seit Mitte der 1980er Jahre mehrheitlich mit Popscores ausgestattet, wobei die üblicherweise männlich konnotierten 
Filmgenres Action-, Abenteuer- und Science-Fiction-Film bemerkenswerter Weise hiervon ausgenommen sind (Kassabian 2001, 69).

\section{Out of Rosenheim (BRD 1987, Percy Adlon)}

Die beiden Hauptpersonen des Films Out of RosENHEIM könnten gegensätzlicher kaum sein: Die bayrische Touristin Jasmin Münchgstettner strandet während einer Amerikareise nach einem Streit mit ihrem Ehemann allein in der Mojave-Wüste. Auf der Suche nach einer Unterkunft landet sie im abgelegenen Motel >Bagdad Café‘, das von der Afroamerikanerin Brenda, die sich gerade von ihrem arbeitsscheuen Ehemann Sal getrennt hat, mehr schlecht als recht unterhalten wird. Die beiden Frauen als Repräsentanten von fast schon resignierten Hausfrauen mittleren Alters raufen sich nach einigen Anlaufschwierigkeiten zusammen und machen das Café durch Showeinlagen mit Zaubertricks und Gesangsauftritten zu einem beliebten Treff von Anwohnern und Fernfahrern.

Beide Frauen werden durch unterschiedliche, von Bob Telson komponierte und Jevetta Steele gesungene Songs charakterisiert: Jasmin ist der 1989 für den Oscar nominierte Titelsong Calling You zugeordnet, eine typische langsame Popballade, die man eigentlich aufgrund der schmerzlich lang ausgehaltenen Töne, der ziellos kreisenden und dissonanzreichen Harmonien und der intim wirkenden Gesangstechnik nah am Mikrophon zur Charakterisierung einer unglücklichen Liebe erwarten würde, die jedoch im Text darauf anspielt, dass Jasmin >wie gerufen $<$ kommt, um einen Wechsel in Brendas privater und beruflicher Situation herbeizuführen (vgl. NB 3). Interessanterweise ertönt mit Ablauf von Jasmins Besuchervisum nur noch die Begleitung des Songs ohne Gesang und verdeutlicht somit die Lücke, die durch Jasmins Abwesenheit entsteht. Brenda und ihre enge Freundschaft und berufliche Partnerschaft mit Jasmin werden dagegen durch einen an Scott Joplin gemahnenden Ragtime vertont, der als traditionell von Schwarzen gepflegtes Musikgenre Brenda gut repräsentiert und zunächst nur in instrumentaler Fassung erscheint, später jedoch auch in einer dem Musical angenäherten Fassung während einer längeren Showeinlage der beiden zum Einsatz kommt und ihren magischen Einfluss auf die Cafégemeinschaft demonstriert (vgl. NB 4). Beide Songs werden vergleichsweise platt kontrastiert: durch aus dem Auto von Jasmins Ehemann ertönende bayrische Volksmusik und den unaufhörlich Johann Sebastian Bachs Präludien übenden Sohn Brendas. 


\title{
NB 3: Text und Akkorde zu Calling You $^{5}$ aus Out of Rosenheim
}

\author{
$\mathrm{Dm} 7 / 5+\mathrm{Eb} 6 / 9 \mathrm{Gm} 7 * \mathrm{C} 7 / 4 \| \mathrm{x} 2$
}

$\operatorname{Dm} 7 / 5+$

Eb6/9

A desert road from Vegas to nowhere

$\mathrm{Gm} 7 *$

$\mathrm{C} 7 / 4$

Some place better than where you've been | A

Dm7/5+

Eb6/9

A coffee machine that needs some fixin'

$\mathrm{Gm} 7 *$

$\mathrm{C} 7 / 4$

In a little Cafe' just around the band

Cm7/5- F Dm7/5- G7

B |

I am calling you - can't you hear me

Em7/5- A7/5+ Dm7/5- G7

I am calling you

$\operatorname{Dm} 7 / 5+\quad$ Eb6/9

A hot dry wind blows right through me

$\mathrm{Gm} 7 *$

$\mathrm{C} 7 / 4$

The baby's cryin' and I can't sleep

$\operatorname{Dm} 7 / 5+$

Eb6/9

But we both know a change is comin'

$\mathrm{Gm} 7 *$

$\mathrm{C} 7 / 4$

Comin' closer so we do release

Cm7/5- F Dm7/5- G7

I am calling you - I know you hear me

Em7/5- A7/5+ Dm7/5- G7

I am calling you

5 http://www.ultimate-guitar.com/tabs/b/bob telson/calling_you_crd.htm (Verifikationsdatum 13.09.07) 
$\begin{array}{lll}\text { Strum } & \text { Gb6 } \mid \text { F7 } \mid \text { Db9 } & \text { Db9-4/A } \mid \text { Db9 } \mid \\ \text { (solo) } & \text { Gb } \mid \text { Db9 } \mid \text { Db9-4/A } \mid \text { Db9 } & \text { Cm7-9+ F7 || }\end{array}$

Cm7/5- F Dm7/5- G7

I am calling you - I know you hear me

Em7/5- A7/5+ Dm7/5- G7

I am calling you

Dm7/5+ Eb6/9

A desert road from Vegas to nowhere

$\mathrm{Gm} 7 * \quad \mathrm{C} 7 / 4$

Some place better than where you've been

Dm7/5+ Eb6/9

A coffee machine that needs some fixin'

$\mathrm{Gm} 7 * \quad \mathrm{C} 7 / 4$

In a little Cafe' just around the band

$\operatorname{Dm} 7 / 5+\quad$ Eb6/9

A hot dry wind blows right through me

$\mathrm{Gm} 7 * \quad \mathrm{C} 7 / 4$

The baby's cryin' and I can't sleep

Dm7/5+ Eb6/9

And I can feel a change is comin'

$\mathrm{Gm} 7 * \quad \mathrm{C} 7 / 4$

Comin' closer so we do release

Cm7/5- F Dm7/5- G7

I am calling you - I know you hear me

Em7/5- A7/5+ Dm7/5- G7

I am calling you 
Kieler Beiträge zur Filmmusikforschung, 2, 2008 / 20

NB 4:

Ragtime Brenda, Brenda ${ }^{6}$ aus Out of RosenheIM
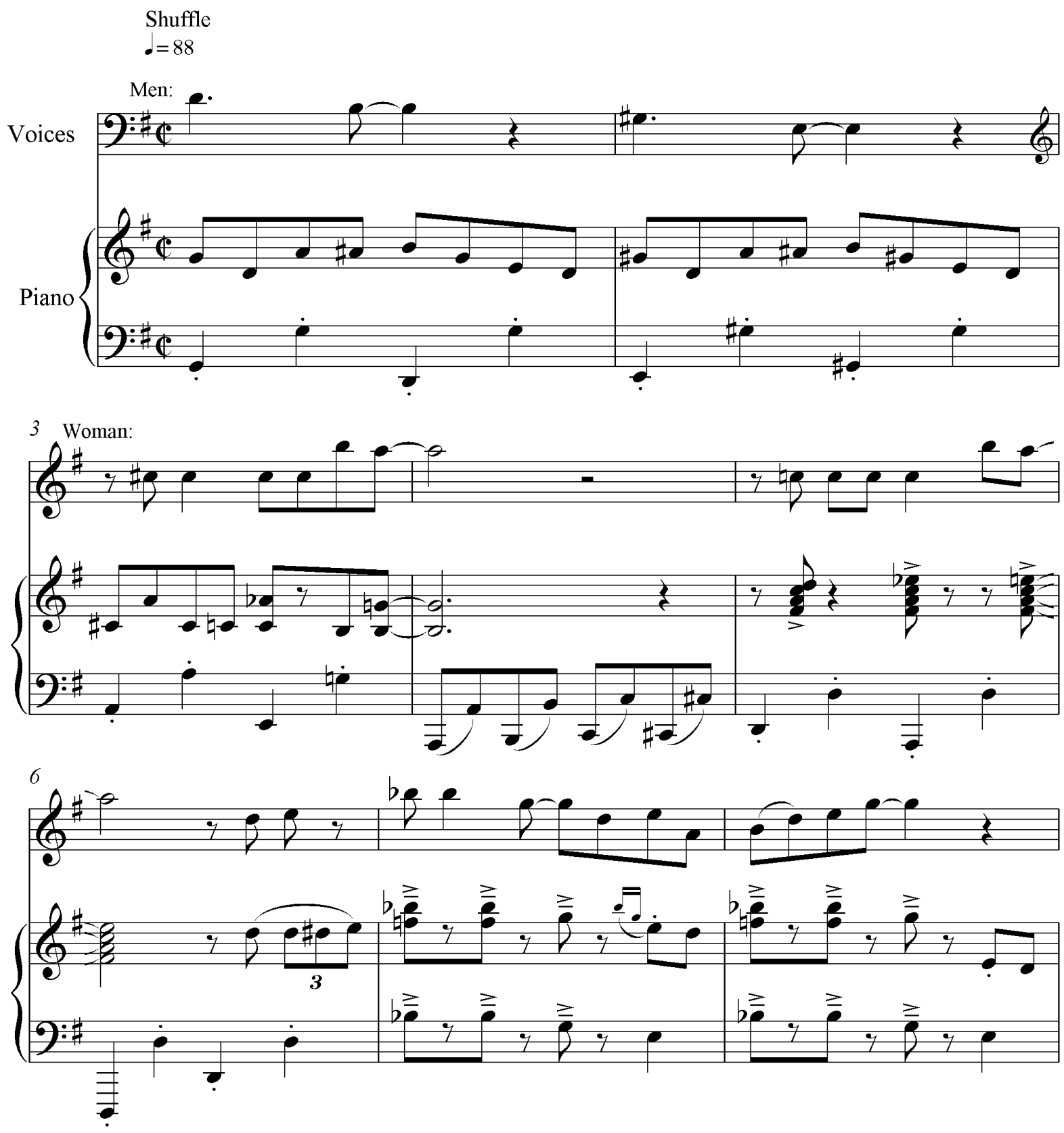


\section{Fried Green Tomatoes (USA 1991, Jon Avnet)}

Im Film Fried Green Tomatoes verschränken sich die Geschichten zweier zeitlich auseinander liegender Frauenfreundschaften: Evelyn Couch, eine von ihrer Ehe enttäuschte Hausfrau in den Wechseljahren, lernt beim Besuch eines Altersheims die Seniorin Ninny Threadgoode kennen und schätzen. Diese erzählt ihr die tragisch-komische Geschichte der innigen Freundschaft zwischen der unangepassten, draufgängerischen und selbstbewussten Idgie und der braven, sanften und sich selbstlos aufopfernden Ruth, die mit Idgies Unterstützung ihren brutalen Ehemann Frank Bennett verlässt und gemeinsam mit Idgie in den Jahren nach der großen Depression in den Südstaten der USA das Whistle Stop Café betreibt. Ninnys Bericht von den zahlreichen gemeinsam bewältigten Problemen (wie z.B. die Anfeindungen durch Mitglieder des Ku-KluxKlans wegen der Anstellung von Afroamerikanern sowie eine fälschliche Anklage Idgies wegen Mordes an Ruths Ehemann) stärken Evelyns Selbstbewusstsein und helfen ihr, ihr Leben neu in den Griff zu bekommen, was auch ihr Ehemann zu spüren bekommt. Der Film erweckt am Ende den Eindruck, Ninny Threadgoode könnte mit der von ihr als Schwägerin beschriebenen Idgie identisch sein.

Die nostalgisch gehaltenen Erinnerungen korrespondieren inhaltlich mit den Gegenwartsszenen, werden jedoch über die Musik gegeneinander abgesetzt: Während die Vergangenheitsszenen im Wesentlichen im traditionellen Hollywoodstil Thomas Newmans $(* 1955)$ vertont werden, herrschen in den Gegenwartsdarstellungen populäre Songs vor, deren Texte teilweise als Kommentare zur Handlung gedeutet werden können. Idgie wird visuell und sprachlich auf männlich konnotierte Verhaltensweisen festgelegt, und die filmische Darstellung spielt mit der häufig in Hollywoodfilmen anzutreffenden Gleichsetzung der „emanzipierten Frau“ mit der „Verbrecherin“. Jedoch kann insgesamt von einer Dekonstruktion des negativen Klischees der starken, emanzipierten Frau gesprochen werden, da die Identifikation mit positiv gezeichneten weiblichen Vorbildern ermöglicht wird (vgl. Steinwidder 2000, insbes. die Tab., 81f). Dies spiegelt sich auch weitgehend in der Vertonung wider, denn hier herrschen von Englischhorn, Streichern und Harfe intonierte Klänge der positiv konnotierten, naturverbundenen Weiblichkeit vor, wenn auch im Verlauf der Geschichte zunehmend eingefügte Blue Notes sowie die bisweilen das Geschehen in den Südstaaten verortenden Bluesklänge eine gewisse moralische Verwerflichkeit und Melancholie andeuten. Insbesondere die erstmalig vollständig gezeigte Bergung von Franks versenktem Kleinlaster wird durch einen geradezu anklagend klingenden Bluesgesang einer Sängerin eingeleitet, und auch den Meineid des Baptistenpredigers im Rahmen der Gerichtsverhandlung, in der Idgie wegen Mordes an Frank angeklagt und aufgrund der Aussage des Geistlichen freigesprochen wird, kommentiert ein gleichzeitig erklingendes Blues-Piano.

Ein im Englischhorn intoniertes Motiv fungiert als >akustischer Trenner (Bullerjahn 2001, 178), der jede Rückblende einleitet, darüber hinaus auch jede emotional bedeutsame Vergangenheitsszene begleitet und somit als idealisierte Zustände und Zeiten kennzeichnet (Flinn 1992, 152). Mit Bekanntwerden des Abrisses 
von Ninnys Haus und beim gemeinsamen Besuch von Ruths Grab durch Evelyn und Ninny hält dieses Motiv sogar Einzug in die Gegenwart, was mit Ninnys Einfluss auf Evelyn korrespondiert.

\section{Thelma \& Louise (USA 1991, Ridley Scott)}

Auch bei der Filmmusik zu Thelma \& Lousse handelt es sich um eine Kombination aus Kompilation und Komposition: Country-, Blues- und Rocksongs überwiegen jedoch und werden durch eine Synthesizer-BluesRock->dramatic score〈 vom deutschstämmigen Hollywoodkomponisten Hans Zimmer $(* 1957)$ ergänzt, die gleichwohl den Showdown beherrscht. Die Unterlegung der Songs erfolgt oft abweichend von filmmusikalischen Traditionen und häufig als $>$ source scoring $\iota^{7}$, d.h. die augenscheinlich in der Filmhandlung erklingende Musik wird dennoch unmerklich dramaturgischen Situationen und Dialogen in der Lautstärke angepasst. Durch eine spezifische Soundmischung sind die Songtexte fast immer hörbar, weshalb sie oft zur Bedeutungsproduktion beitragen können. Jedoch haben die Songs wie bei vielen Pop-Soundtracks auch oft Zufallsqualität und stehen in sehr vagen Zusammenhalt mit dem Visuellen, häufig sogar nur mit dem Beginn des Songs (Kassabian 2001, 79f).

Im Mittelpunkt der Story stehen zwei Freundinnen, die Hausfrau Thelma und die Serviererin Louise, die zu einem unbeschwerten Wochenendtrip in die Natur aufbrechen, um ein paar Tage ohne Männer in einer Hütte am See zu verbringen. Sie kommen nie an, denn bereits die erste Nacht birgt unerwartete Probleme: Louise rettet ihre Freundin Thelma vor einer Vergewaltigung auf dem Parkplatz vor einer Musikkneipe, indem sie den Täter, der sie provoziert, erschießt, wodurch sich beide zu einer Flucht vor Polizei und FBI genötigt sehen. Hat es zunächst den Anschein, als strebten sie zunehmend kompromisslos ihre Freiheit und eine Emanzipation von der Männerwelt an $^{8}$, so dringen sie im Gegenteil in diese ein und adaptieren sich, indem sie deren Regeln und Gesetze übernehmen, wozu neben rüden Sprüchen und Waffengebrauch auch der offensive Umgang mit der eigenen Sexualität gehört. Der Film bedient sämtliche Klischees des Road Movies, wie z.B. die grandiosen Landschaftsaufnahmen, die die räumliche Veränderung dokumentieren, und die mit Songs musikalisierte `Freiheit der Straße`, mit dem bedeutsamen Unterschied, dass die Akteure weiblich sind, was häufig zu Situationskomik führt.

Der Gesinnungswandel der beiden Frauen zeigt sich auch in der Filmvertonung: Bevor Thelma und Louise die Musikkneipe erreichen, also vor ihrem Eindringen in die Männerwelt, erklingen im Soundtrack fünf

7 Earle Hagen definiert in Scoring for Films. A Complete Text (New York 1971, S. 200) >source scoring< in Abgrenzung zu >source music < und >pure < oder >dramatic scoring ‘ folgendermaßen: »This kind of music is like source in its content, but tailored to meet scoring requirements. [...] This kind of cue can start as pure source music and change over to source scoring. The main difference between Source and Source Scoring is that source scoring takes on a much closer relationship to the film. It follows the framework of the scene more critically and matches the nuances of the scene musically. It is closer to scoring in intent, but related to source in content."

8 Vgl. eine diesbezügliche Analyse von Frank Hörnlein 1996. 


\section{Kieler Beiträge zur Filmmusikforschung, 2, 2008 / 23}

ausschließlich von Frauen gesungene Songs. Dies ändert sich erst bei Thelmas Tanz mit ihrem aufdringlichen >Verehrer`, da hier die Live-Rockband des Clubs mit ihrem männlichen Sänger ins Sicht- und Hörfeld rückt. Als die Frauen nach der vereitelten Vergewaltigung den Parkplatz fluchtartig verlassen und kurz anhalten, ertönt aus dem Musikautomat des Kaffeeladens Tammy Wynettes Song I Don't Wonna Play House, womit schon an dieser frühen Filmstelle abgedeutet wird, dass Thelma und Louise nie wieder an Heim und Herd zurückkehren werden (Kassabian 2001, 80f). In der Anfangssequenz werden die Protagonistinnen noch als recht gegensätzlich etabliert: Während die kesse und selbstbewusste Louise eine Atmo aus lauter CountryMusik und Restaurant-Stimmgewirr umgibt, charakterisieren die devote und unselbstständige Thelma leise Geräusche des häuslichen Küchenfernsehers. Geschirrklappern kennzeichnet jedoch beide Räume und fungiert als verbindendes akustisches Element einer weiblichen Lebenswelt, womit die vor allem auf optischer und Handlungsebene postulierten Unterschiede unterwandert werden (Flückiger 2001, 328f).

Durch die Verwendung populärer Musik verankert der Film die Narration im Alltag, denn es handelt sich um Musik, die teilweise allbekannt ist, jederzeit aus dem Radio ertönen könnte und auch häufig in THELMA \& LouIse aus dem Autoradio erklingt. Das Auto mit seiner Musik - im Road Movie ein gewöhnlich männlich bestimmter Raum - fungiert als wieder gewonnener geschützter Raum, in dem einem sogar das Mitgrölen nicht peinlich ist. Auch die zu Beginn sehr zurückhaltende dramatic score von Zimmer passt sich durch die Verwendung von Slide Guitar, Mundharmonika und Banjo den häufigen Countrysongs an und entspricht zudem dem Musikklischee für die unendlichen Weiten des amerikanischen Westens. Mit Mexiko als neuem Fernziel tauchen zusätzlich Mariachi-Trompetenfiguren auf und verheißen eine Zukunft in Mexiko, die es nicht geben wird (vgl. Flückiger 2001, 82-84). Hinzu treten im weiteren Verlauf des Films zunehmend verhallte, bedrohlich wirkende Tomtoms. Die zum Showdown hin immer dominantere, stärker pulsierende, dichter instrumentierte, gleichwohl von einer verhallten Mundharmonika angeführte dramatic score mündet bei der gemeinsam beschlossenen finalen Fahrt auf den Abgrund des Grand Canyons zu in positiv gestimmte Gospelklänge, womit die beiden Frauen zu Heldinnen, wenn nicht gar zu Märtyrerinnen stilisiert werden.

\section{Resümee}

»Musik teilt die Frauen in Gefährliche und Zahme, macht aus der guten Frau eine langweilig-ebenmäßige und aus der Erotischen eine mit Konfliktpotential angereicherte Person. Sie idealisiert die fürsorgend-empathische Frau. Aber sie berührt auch eine andere Ebene, indem beispielsweise die Musik, die die sexuell begehrende Frau beschreibt, zugleich faszinierend wirkt $[\ldots] . \ll($ Rieger 1996, 234)

Rieger stellt resümierend am Ende ihres Buches zu Filmmusiken in Filmen Alfred Hitchcocks fest, dass die Filmmusikkomponisten über fünf Jahrzehnte hinweg »trotz ihrer stilistischen Eigenarten traditionell an das 19. Jahrhundert gebunden [waren], das musikalischen Geschlechter-Typisierungen unterlag« (Rieger 1996, 225). Hierbei muss angemerkt werden, dass der auf Unterhaltung und Kassenerfolg abzielende Spielfilm für 


\section{Kieler Beiträge zur Filmmusikforschung, 2, 2008 / 24}

das verlässliche Einplanen von Wirkungen auf das Abrufen von Klischees vertrauen muss und somit auf die Verwendung prototypischer Musik angewiesen ist. Häufig ist ein Liebesthema zentral, das das Begehren des Mannes illustriert und somit nur indirekt an die Frau gekoppelt ist.

»Die Mittel, die romantische Liebe beschreiben, ähneln sich: Streicher (sordino)-Besetzung, Seufzermotive, kleinschrittige legato-Melodien, piano-Dynamik. Bevorzugt werden Streicher, Celesta, Flöte und Harfe. Zuweilen findet sich ein Tritonus in der Melodie, um bevorstehende Gefahren oder charakterliche Defizite vorwegzunehmen« (Rieger 1996, 231)

Bezüglich Riegers letzter Aussage ist meines Erachtens eine Einschränkung angebracht: Zwar wird der Tritonus häufig mit der femme fatale sowie sonstigen mehr oder weniger bösartigen und grausamen Frauengestalten und ihren moralisch verwerflichen Handlungen verknüpft, jedoch ist dies kein Alleinstellungsmerkmal: Mindestens genauso häufig ist er auch zentral bei der musikalischen Charakterisierung psychisch-kranker und -labiler Personen jedweden Geschlechts und sämtlicher männlicher Bösewichte und Teufelsinkarnationen. Man kann sogar insgesamt feststellen, dass die Kennzeichen des musikalisch Bösen weitgehend gender-unabhängige Verwendung finden. Allerdings ist Rieger sicherlich zuzustimmen, dass die Geschlechter traditionell »musikalisch ungleichgewichtig inszeniert« (Rieger 1996, 233) werden, jedoch gilt dies gleichermaßen für die visuelle und sprachliche Ebene und somit für die gesamte Narration, die letztlich immer ein Spiegel gesellschaftlicher Verhältnisse ist.

In den analysierten älteren Filmen wird die Emanzipation von Frauen mit charakterlichen Schwächen gekoppelt und damit negativ dargestellt: So kann man die Kettenraucherin und Hosenträgerin Rebecca durchaus als Modell einer emanzipierten Frau sehen, die furchtlos, intelligent und selbstständig ist und ihr Sexualleben selbst bestimmt. Jedoch wird sie zusätzlich als charakterlich defizitär charakterisiert, denn sie agierte offensichtlich egoistisch und skrupellos und instrumentalisierte für sich andere Menschen, die sie zugleich verachtete. Der durch Maxim de Winter repräsentierte bürgerliche Mann bevorzugt die zuverlässige, langweilige und sich unterordnende Kindfrau, wenn er sich auch nach der sexuell reifen Frau sehnt. In den ausgewählten neueren Filmen erhält die Frauenemanzipation dagegen eine positive Aufwertung, was sich auch in den Filmmusiken widerspiegelt: Die Filmheldinnen werden sympathisch charakterisiert, und Männer kommen insgesamt eher schlecht weg. Jedoch wird diese Freiheit teilweise dadurch erkauft, dass die Frauen doch ihre >gerechte Strafe` erhalten - auch Idgie wählt durch Heirat schließlich eine bürgerliche Existenz, wird jedoch mit einem geistig behinderten Sohn für ihr Vorleben 〉bestraft«, und Thelma und Louise agieren wie männliche Outlaws und wählen den Freitod. Selbst die deutsche Touristin Jasmin darf am Film-Ende nicht unbeschwert ihre gemeinsame Freiheit mit der schwarzen Motel- und Cafébesitzerin Brenda genießen, denn der Maler Rudi Cox macht ihr einen Heiratsantrag und Brenda nimmt ihren Ehemann wieder bei sich auf. 
Kieler Beiträge zur Filmmusikforschung, 2, 2008 / 25

\section{Literatur}

Bullerjahn, Claudia (2001) Grundlagen der Wirkung von Filmmusik. Augsburg: Wißner (Wißner Lehrbuch. 5.).

Bullerjahn, Claudia (2005) Analyse von Filmmusik und Musikvideos. In: Qualitative Medienforschung. Ein Handbuch. Hrsg. v. Lothar Mikos u. Claudia Wegener. Konstanz: UVK, S. 484-495.

Fabich, Rainer (1993) Musik für den Stummfilm. Analysierende Beschreibung originaler Filmkompositionen. Frankfurt [...]: Peter Lang (Europäische Hochschulschriften: Musikwissenschaft. 94.).

Flinn, Caryl (1986) The >Problem^ of Feminity on Theories of Film Music. In: Screen 27,6, S. 56-82.

Flinn, Caryl (1992) Strains of Utopia. Gender, Nostalgia, and Hollywood Film Music. Princeton: Princeton University Press.

Flückiger, Barbara (2001) Sound Design. Die virtuelle Klangwelt des Films. Marburg: Schüren.

Hagen Earle (1971) Scoring for Films. A Complete Text. New York: Criterion Books.

Hörnlein, Frank (1996) Thelma \& Louise: Neue Angebote für Identifikation und Konstruktion des Geschlechterverhältnisses im Spielfilm? In: Sinnwelt Film - Beiträge zur interdisziplinären Filmanalyse. Hrsg. v. Wilhelm Hofmann. Baden-Baden: Nomos, S. 69-104.

Kalinak, Kathryn (1982) The Fallen Women and the Virtuous Wife: Musical Stereotypes in The Informer, Gone With the Wind, and Laura. In: Film Reader 5, S. 76-82.

Kassabian, Anahid (2001) Hearing Film. Tracking Identifications in Contemporary Hollywood Film Music. London: Routledge.

Lissa, Zofia (1965) Ästhetik der Filmmusik. Berlin: Henschelverlag.

Rapée, Ernö (1970) Motion Picture Moods for Pianists and Organists. New York: Arno Press. Reprint d. Erstausgabe 1924.

Rieger, Eva (1996) Alfred Hitchcock und die Musik. Eine Untersuchung zum Verhältnis von Film, Musik und Geschlecht. Bielefeld: Kleine.

Steinwidder, Patrick (2000) (W)Er hat Angst vor starken Frauen? Identifikationsangebote in GrüNE Tomaten. In: Medien-Impulse 32,8, S. 73-83.

Tagg, Philip (2006) Music, Moving Images, Semiotics and the Democratic Right to Know. In: Music and Manipulation: On Social Uses and Social Control of Music. Ed. By Steven Brown \& Ulrik Volgsten. New York/Oxford: Berghahn Books, S. 163-186.

Tagg, Philip / Clarida, Bob (2003) Ten Little Title Tunes. Towards a musicology of the mass media. New York [...]: The Mass Media Music Scholar's Press. 
Kieler Beiträge zur Filmmusikforschung, 2, 2008 / 26

\section{Empfohlene Zitierweise:}

Claudia Bullerjahn: Gender-Konstruktion durch Filmmusik. Eine analytische Betrachtung am Beispiel der Vertonung von Frauenfiguren in Filmen von Alfred Hitchcock und im neueren Frauenfilm

In: Kieler Beiträge zur Filmmusikforschung 2, 2008.

URL: http://www.filmmusik.uni-kiel.de/beitraege.htm

Datum des Zugriffs: 1.10.2008.

Kieler Beiträge für Filmmusikforschung (ISSN 1866-4768)

Copyright (C) by Claudia Bullerjahn. All rights reserved.

Copyright (C) für diese Ausgabe by Kieler Gesellschaft für Filmmusikforschung. All rights reserved.

This work may be copied for non-profit educational use if proper credit is given to the author and „Kieler Beiträge für Filmmusikforschung“" 